\title{
Structure-function clustering in multiplex brain networks
}

\author{
J. J. Crofts ${ }^{1}$, M. Forrester ${ }^{2}$ and R. D. O’DeA ${ }^{2}$ \\ 1 Nottingham Trent University - School of Science and Technology, Nottingham, NG11 8NS, UK \\ 2 University of Nottingham - Centre for Mathematical Medicine and Biology, School of Mathematical Sciences, Not- \\ tingham, NG7 2RD, UK
}

PACS 87.18.Sn - Neural networks

PACS 89.75.-k-Complex systems

PACS 87.18.Vf - Systems biology

\begin{abstract}
A key question in neuroscience is to understand how a rich functional repertoire of brain activity arises within relatively static networks of structurally-connected neural populations: elucidating the subtle interactions between evoked 'functional connectivity' and the underlying 'structural connectivity' has the potential to address this. These structural-functional networks (and neural networks more generally) are more naturally described using a multilayer or multiplex network approach, in favour of standard single-layer network analyses that are more typically applied to such systems. In this letter, we address such issues by exploring important structure-function relations in the Macaque cortical network by modelling it as a duplex network that comprises an anatomical layer, describing the known (macro-scale) network topology of the Macaque monkey, and a functional layer derived from simulated neural activity. We investigate and characterize correlations between structural and functional layers, as system parameters controlling simulated neural activity are varied, by employing recently described multiplex network measures. Moreover, we propose a novel measure of multiplex structure-function clustering which allows us to investigate the emergence of functional connections that are distinct from the underlying cortical structure, and to highlight the dependence of multiplex structure on the neural dynamical regime.
\end{abstract}

Introduction. - Network science provides a powerful set of tools for studying the complex systems that arise throughout the biological, physical, social, and information sciences [1]. A defining feature of such systems is that of emergence, i.e. the collective dynamics of such systems are often poorly predicted by the dynamics of any single node. Thus, understanding the impact of complex network topology on the dynamic processes that these structures sustain is of fundamental importance. Neuroscience, with its heterogeneous, multiscale patterns of neural connectivity and rich dynamic repertoire, provides a paradigmatic model in which to study dynamic processes on complex networks. Synchronisation and propagation behaviour of neural activity, for example, is considered to be a key mechanism for many neural processes, and has been studied intensively in both idealised network structures [2-4] and, more recently, within physiologically inspired networks such as cortical networks of the rat [5], cat $[6,7]$, and Macaque monkey [8] and humans $[9,10]$.

An alternative approach to understand the influence of network structure is to consider the interactions between the so-called 'functional connectivity' network, that is evoked by dynamic processes occurring between structurally connected neural populations [11], and the underlying anatomical connectivity network. Functional connectivity refers to networks inferred from the synchronisation of neural activity between brain areas (typically obtained experimentally via blood oxygenation leveldependent (BOLD) functional MRI (fMRI), or coherence in electro- or magnetoencephalogram (EEG/MEG) signals), as opposed to structural connections observed via in vivo neuroimaging (typically corresponding to long-range fibre bundles). The predominance of complex network research within this area has so far focussed on summarising functional and structural networks in isolation, successfully revealing many common features such as smallworldness [12], the existence of hubs [13], and a hierarchically modular structure [14]. Importantly, a number of recent studies have shown that brain injury and disease manifest via faulty, disrupted brain networks of both 
structure [15-18] and function [19-22]; more widely, a fundamental question is to elucidate how the relatively static anatomical connectivity of the brain supports a vast functional repertoire of higher brain functions, such as action, perception and cognition [23]. Improved understanding of the link between anatomical and functional brain networks has the potential to address this and yet, despite significant progress in our understanding of human brain connectivity over the past decade, this relationship remains poorly understood (see, e.g., [24,25] and references therein).

This letter builds upon recent theoretical advances in complex networks that allows for the treatment of two or more interacting network structures simultaneously [26-28]. Specifically, we employ multiplex network models to analyse simultaneously anatomical and functional brain networks, thereby gaining additional insights into important structure-function brain relationships via emergent multiplex network properties. In particular, we employ a multiplex approach to analyse structure-function relations, extending [8] to describe the relationship between a physiologically relevant structural network (obtained from the CoComac database [29]) and functional connections derived from simulated neural activity of a set of neural subunits, whose connectivity is defined by the aforementioned structural information. Under systematic variation of the model parameters, we investigate the evoked changes in functional network topology in terms of the structure-function relationships revealed by relevant multiplex measures. In addition, we propose a novel measure of multiplex structure-function clustering in order to describe the emergence of functional connections between brain regions in the absence of direct anatomical links. Such a divergence between functional and structural networks is fundamental to the brain's wide functional repertoire; our new quantification of this within a multiplex framework and, in particular, our proposed new clustering measure which seeks to measure precisely this disparity, represents a new avenue towards understanding structurefunction relationships at a more fundamental level.

Multiplex brain setup. - A general multiplex network comprises $N$ nodes, connected via edges belonging to the $M$ layers. Such a multiplex is fully specified by the vector $\mathbf{A}=\left[A^{[1]}, \ldots, A^{[M]}\right]$ whose entries $A^{[\alpha]}=\left\{a_{i j}^{\alpha}\right\}$ are matrices with non-zero entries in the $i j$ th position if node $i$ connects to node $j$ in layer $\alpha$, otherwise $\left\{a_{i j}^{\alpha}\right\}=0$. The degree vector, $\mathbf{k}(i)$, naturally extends the notion of network degree to the multiplex setting such that its entries $k^{[\alpha]}(i)=\sum_{j} a_{i j}^{[\alpha]}$ give the standard network degree of node $i$ restricted to layer $\alpha$, and with obvious generalisation to a directed multiplex with in- and out-degree vectors having entries given by $k_{\text {in }}^{[\alpha]}=\sum_{j} a_{j i}^{[\alpha]}$ and $k_{\text {out }}^{[\alpha]}=\sum_{j} a_{i j}^{[\alpha]}$ respectively.

Herein, we consider only two-layer networks (duplexes) in which the structural network (layer 1) is given by the known cortical network of the Macaque monkey and the

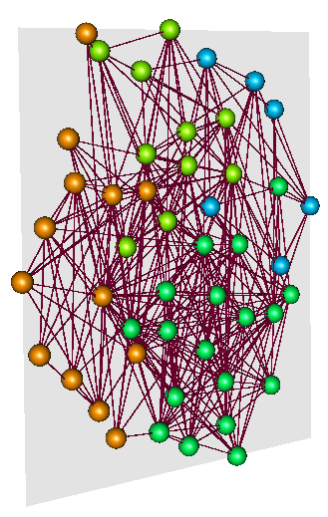

(a) Structure

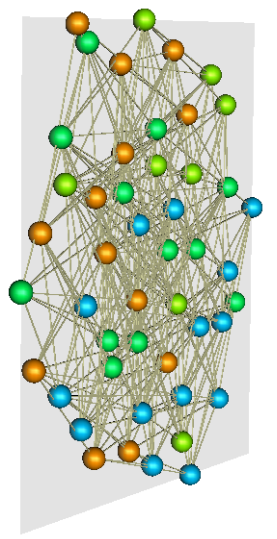

(b) Function
Fig. 1: An example multiplex brain network, indicating (a) the underlying structural network (the known cortical network of the Macaque monkey) and (b) a functional network derived from synchronisation of neural populations governed by (1).

functional network (layer 2) is derived by measuring pairwise correlations between time series of simulated neural activity on all nodes (representing cortical areas). Figure 1 shows an example multiplex brain network constructed in this way. More specifically, we use a parcellation of the Macaque cortex consisting of 47 brain regions which are linked by 505 directed fibres [29], yielding a binary connectivity matrix $A^{[1]}$ such that $\left\{a_{i j}^{[1]}\right\}=1$ if brain region $i$ projects onto brain region $j$, and is otherwise zero.

To determine $A^{[2]}$, each cortical area is modelled by a Wilson-Cowan [30] node of two interacting populations of neurons:

$$
\begin{aligned}
& \frac{\mathrm{d} u_{i}}{\mathrm{~d} t}=-u_{i}+f\left(c_{1} u_{i}-c_{2} v_{i}+P+\epsilon \sum_{j} w_{i j}^{[1]} u_{j}\right) \\
& \frac{\mathrm{d} v_{i}}{\mathrm{~d} t}=-v_{i}+f\left(c_{3} u_{i}-c_{4} v_{i}+Q\right) .
\end{aligned}
$$

Here, $u_{i}(t)$ denotes the activity of the population of excitatory neurons within region $i$, and $v_{i}(t)$ the activity of inhibitory neurons. The population firing rate is given by the sigmoid $f(x)=1 /(1+\exp (-x))$; interconnectivity is encoded by the matrix $w_{i j}=a_{i j}^{[1]} / \sum_{k} a_{i k}^{[1]}$, in which entries of $a_{i j}^{[1]}$ are normalised in order that each node receives comparable input. The global between-node connectivity strength is controlled by the parameter $\epsilon$ which we henceforth set to unity; for $\epsilon=0$ the network decouples, with node dynamics determined only by the parameter values and initial data. The constants $c_{1}, \ldots, c_{4}$ define the strength of within-node interactions between neural populations and are chosen to be $c_{1}=c_{2}=c_{3}=10$ and $c_{4}=-2$ as in [31]. The remaining parameters, $P$ and $Q$, are control parameters that represent the basal input to each population. Equation (1) represents a simple (but historically important) model of the oscillatory behaviour of interacting neural populations. More sophis- 


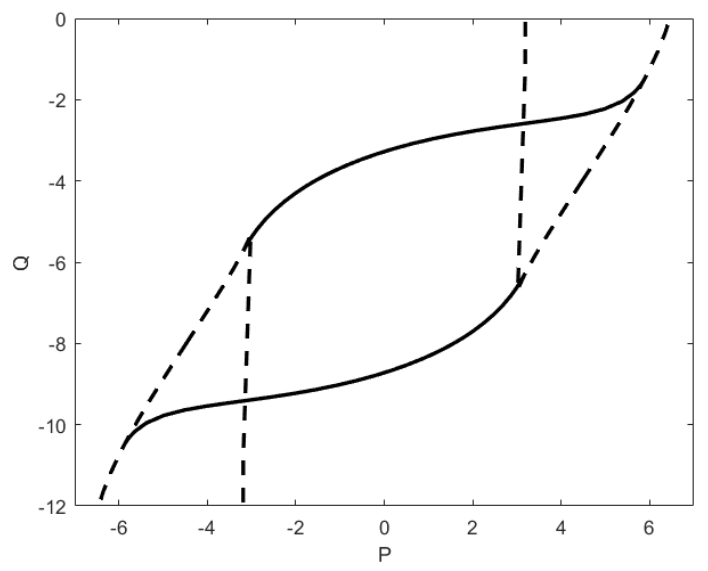

Fig. 2: Bifurcation sets of a single Wilson-Cowan node for $c_{1}=$ $c_{2}=c_{3}=10$ and $c_{4}=-2$. The dashed curve is the saddlenode bifurcation set, the solid curve is the Hopf bifurcation set.

ticated neural mass models that more faithfully reproduce observed EEG and fMRI data (see, e.g., [32-34] and references therein) may be employed with little difficulty; however, our focus is to interrogate the relationship between the underlying structural network and the evoked functional connections, rather than on detailed analysis of neural population dynamics. The model (1) supports transition between trivial steady-state dynamics and oscillatory neural-like behaviour via a Hopf bifurcation. A skeleton of the bifurcation diagram for a single Wilson-Cowan node is displayed in Figure 2, highlighting, in particular, the Hopf bifurcation set (see [31] for a detailed bifurcation analysis). In view of the important role of rhythmic brain activity in neural processing we study only parameter choices that support oscillations. The model (1) supports transition between trivial steady-state dynamics and oscillatory neural-like behaviour under variation of $P, Q$; in view of the important role of rhythmic brain activity in neural processing we study only parameter choices that support oscillations.

The functional network is derived from the simulated neural activity by calculating pairwise the Pearson's correlation coefficient of the time series of all cortical areas. The resulting correlation matrix was binarised (with threshold chosen such that the resulting functional network is of equal density to the underlying structural network) to obtain an undirected functional connectivity network describing cortical areas with statistically similar activity. In view of the nonlinear oscillations supported by (1), we also investigated functional networks arising from consideration of the mean phase coherence [35], observing qualitatively similar results (as in [8]) which are therefore not included. We remark that more advanced measures may be employed to derive a directed 'effective connectivity' structure; however, here we concentrate on undirected functional networks for consistency with similar studies in the literature, and comparability with [8], in particular.

To highlight the influence of the specific topology of the macaque cortical network we compute normalised multiplex measures obtained by dividing values computed for the structure-function duplex by the corresponding ensemble averages obtained from random surrogates. These surrogate duplexes are constructed by simulating neural activity on 100 directed random networks with in- and outdegree sequences matching that of the structural layer.

Multiplex measures. - To characterise the structural properties of the duplex brain network we consider recently forwarded multiplex measures [26-28] and, moreover, propose a novel adaptation of particular relevance to structure-function relationships in neural systems.

Firstly, we quantify the extent to which the link structure varies between the two layers by considering the global overlap, $\mathcal{O}$, given by

$$
\mathcal{O}=\sum_{i \neq j} a_{i j}^{[1]} a_{i j}^{[2]} .
$$

Secondly, we consider the multiplex clustering coefficient, in view of the importance of clustering in smallworld and hierarchically modular phenomena widely observed in both empirical and theoretical analyses of neural networks. Recall that the standard (single-layer) local clustering coefficient, $c(i)$, of node $i$ is given by the ratio of the number of triangles (or 3-cycles) including node $i$ to the number of connected tuples centered on node $i$. Multiplex networks, however, contain more general 3-cycles that traverse multiple layers. Noting that we restrict here to two-layer multiplexes, clustering on each node is given as $[27]$

$$
C(i)=\frac{\sum_{j \neq i, m \neq i}\left(a_{i j}^{[1]} a_{j m}^{[2]} a_{m i}^{[1]}+a_{i j}^{[2]} a_{j m}^{[1]} a_{m i}^{[2]}\right)}{k_{i}^{[1]}\left(k_{i}^{[1]}-1\right)+k_{i}^{[2]}\left(k_{i}^{[2]}-1\right)}
$$

which may be more succinctly expressed

$$
C(i)=\frac{\left(A^{[1]} A^{[2]} A^{[1]}+A^{[2]} A^{[1]} A^{[2]}\right)_{i i}}{k_{i}^{[1]}\left(k_{i}^{[1]}-1\right)+k_{i}^{[2]}\left(k_{i}^{[2]}-1\right)} .
$$

The average clustering coefficient for the network is given by $\mathcal{C}=(1 / N) \sum_{i} C(i)$. Note that both these measures, as defined in [27], assume that intra-layer adjacency relations are symmetric $\left(\right.$ i.e. $A^{[\alpha]}=A^{[\alpha]^{T}}$ ) thus we ignore directionality of the structural layer when computing (2) and (3).

In addition to these standard measures we consider a restriction of (3) such that triads comprise a structural tuple, closed by a functional edge, in the absence of a structural one. Such a clustering is pertinent to the emergence of functional connections that arise between structurally unconnected regions in neural systems. Moreover, we incorporate the directionality of the structural layer thus enabling us to consider the influence of structural patterns (i.e. 3-node motifs) on the observed synchronisation dynamics. This is given by 


$$
\begin{aligned}
\widetilde{C}(i) & =\frac{\sum_{j \neq i, m \neq i}\left(a_{i j}^{[1]}+a_{j i}^{[1]}\right) a_{j m}^{[2]}\left(a_{m i}^{[1]}+a_{i m}^{[1]}\right)\left(1-a_{j m}^{[1]}\right)\left(1-a_{m j}^{[1]}\right)}{\left(k_{\mathrm{tot}}^{[1]}(i)\left(k_{\mathrm{tot}}^{[1]}(i)-1\right)-2\left(A^{[1]}\right)_{i i}^{2}\right)\left(1-c_{\mathrm{all}}^{[1]}(i)\right)} \\
= & \frac{\left(\left(A^{[1]}+A^{[1]^{T}}\right)\left(A^{[2]} \circ\left(E-A^{[1]}\right) \circ\left(E-A^{[1] T}\right)\right)\left(A^{[1]}+A^{[1]^{T}}\right)\right)_{i i}}{\left(k_{\mathrm{tot}}^{[1]}(i)\left(k_{\mathrm{tot}}^{[1]}(i)-1\right)-2\left(A^{[1]}\right)_{i i}^{2}\right)\left(1-c_{\mathrm{all}}^{[1]}(i)\right)}
\end{aligned}
$$

where $\circ$ denotes element-wise multiplication, $k_{\text {tot }}^{[1]}(i)$ and $c_{\text {all }}^{[1]}(i)$ denote respectively, the total degree (i.e. the sum of the in- and out-degrees) and the directed clustering coefficient, as defined in [36], for the $i$ th structural node. The factor $\left(1-c_{\text {all }}^{[1]}(i)\right)$ ensures that $\widetilde{C}(i) \in[0,1]$, while $\left(1-a_{j m}^{[1]}\right)$ and $\left(1-a_{m j}^{[1]}\right)$ terms prohibit triads that are closed both in the structural and functional layers. The global version of Equation (4) is $\widetilde{\mathcal{C}}=(1 / N) \sum_{i} \widetilde{C}(i)$.

Our definition treats all possible directed triangles equivalently. Of particular interest here, however, are the distinct network properties that emerge by consideration of different structural connectivity motifs since they have been shown to both enrich and diversify the dynamical landscape of synchronised cortical states [37]. Analogously to [36] these may be characterised by considering a single node $k$, with neighbours $i, j$; in the duplex under consideration these are (i) cycles: a directed structural connection in layer 1 between $i$ and $j$ via $k$, closed by a functional edge in layer 2 ; and (ii) inward (outward): $k$ holds two structural inward (outward) edges.

In view of our current focus on understanding structurefunction relationships in a neural context, only three of the four possible triangles are of interest: cycles (of which there are two) have intuitive meaning in the context of indirect functional connectivity, whereas outward tuples correspond to the population synchrony that arises in the presence of common drive; inward structures will not be considered. Table 1 illustrates the relevant triads, together with formulæ for the corresponding clustering coefficient.

Results. - Figure 3 compares the multiplex network measures in (2) and (3) with standard normalised singlelayer clustering in the functional layer, for a range of choices of $P$, and $Q$. Here and throughout, presented results depict measures normalised relative to that observed in a random graph (i.e. $\mathcal{O} /\left\langle\mathcal{O}_{\text {rand }}\right\rangle$ and $\left.\mathcal{C} /\left\langle\mathcal{C}_{\text {rand }}\right\rangle\right)$. In all cases we observe a region of $(P, Q)$-space in which each measure is elevated. There are two principal features: a central rectangular region, and a pair of branched structures. The boundary reflects closely the bifurcation structure of (1) corresponding to an uncoupled Wilson-Cowan node (in the uncoupled case $\epsilon=0$ ), at which oscillatory solutions are created; the reduced overlap and clustering observed between the rectangular and branched structure arises at values of $P$ and $Q$ at which reduced synchrony occurs in oscillatory node dynamics [8]. Importantly, we observe in general a strong resemblance between structural and functional networks, and high clustering, when the dynamics are close to a phase transition, in agreement with recent empirical $[38,39]$ and computational $[25,40]$ studies suggesting that structure-function relations are heightened as system dynamics approach criticality. Comparison of Figure 3(a) and (c), however, indicates significant differences between single and multilayer clustering, elevated values of the latter extending over a wider region of parameter space and, in particular, being less tightly constrained to critical transition regions. These results highlight that important additional information regarding emergent structure-function relations is revealed by considering networks simultaneously.

In figure 4 we consider measures of particular relevance to emergent structure-function relations within neural networks, as defined in Table 1. Figure 4(a) illustrates our new measure $\widetilde{\mathcal{C}}^{\text {both }}$ (averaged over all nodes and normalised), indicating considerable additional detail in comparison to Figure 3(c). Reminiscent of that shown in Figure 3(a), our new measure reflects less tightly the criticality structure observed in Figures 3(b,c). Figures 4(b,c) indicate the individual contributions to $\widetilde{\mathcal{C}}^{\text {both }}$ from the different network motifs under consideration (see Table 1), highlighting that the kind of structure-function relationships that emerge depend strongly on the dynamical regime under consideration. In particular, we observe distinct areas of parameter space in which clustering is dominated by either common drive or indirect functional connectivity. Comparison with Figure 3(b) shows that when structural and functional networks show heightened correspondence, $\widetilde{\mathcal{C}}^{\text {both }}$ is dominated by common drive, and that this is especially the case within the transition regions at the boundary. The converse is true in the intermediate regions of lower synchrony (in [8] these regions were highlighted as those for which synchronous solutions are unstable in a weakly-coupled network), in which we infer that the interplay between neural dynamics and the particular network structure supports complex patterns of partial synchrony that manifest as emergent functional connections. Numerical results, corresponding to both single node dynamics and in the connected network (that are 


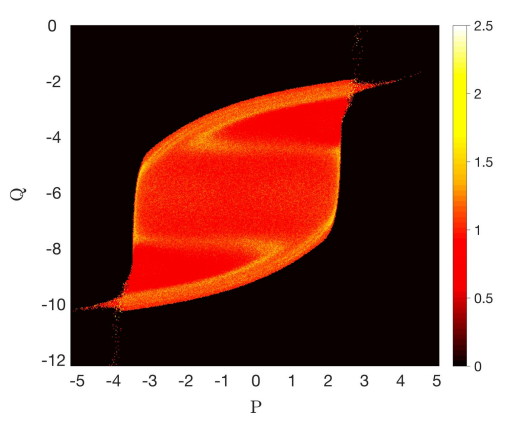

(a)

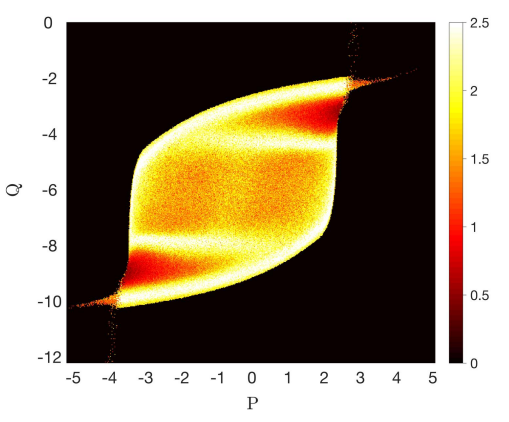

(b)

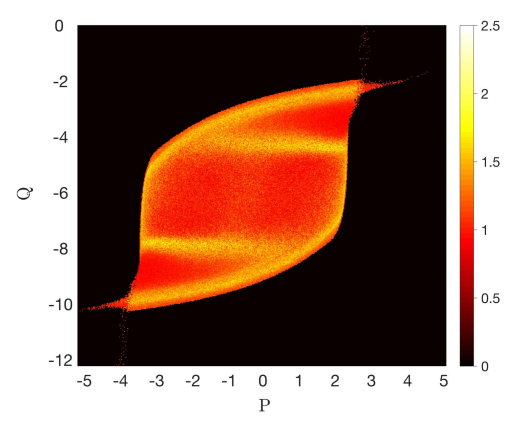

(c)

Fig. 3: Comparison of multiplex vs. single-layer measures as a function of the basal activation parameters $P$ and $Q$ of the Wilson-Cowan model (1). (a) Standard clustering of the functional layer, (b) global overlap, and (c) multiplex clustering. All measures are normalised against random surrogates; see text.

Table 1: Patterns of inter-layer triangles comprising directed structural tuples closed by an undirected functional edge, and their associated clustering coefficients. Here, o denotes element-wise matrix multiplication, $k_{\text {in }}^{[1]}(i)$ and $k_{\text {out }}^{[1]}(i)$ the in- and out-degree of the $i$ th node in layer one respectively, and $c_{\#}^{[1]}(i)$, with $\{\#\} \in\{$ cycle, out, both $\}$, the directed, single-layer clustering coefficient as defined in [36]. Global measures are obtained by averaging over all nodes $\left(\right.$ i.e. $\left.\widetilde{\mathcal{C}}^{\#}=1 / N \sum_{i} \widetilde{C}^{\#}(i)\right)$.

Patterns
Cycle
Both $\widetilde{C}^{\text {cyc }}(i)=\frac{\left(A^{[1]}\left(A^{[2]} \circ\left(E-A^{[1]}\right) \circ\left(E-A^{[1] T}\right)\right) A^{[1]}\right)_{i i}}{\left(k_{\text {in }}^{[1]}(i) k_{\text {out }}^{[1]}(i)-\left(A^{[1]}\right)_{i i}^{2}\right)\left(1-c_{\text {cyc }}^{[1]}(i)\right)}=\frac{\left(A^{[1]}\left(A^{[2]} \circ\left(E-A^{[1]}\right) \circ\left(E-A^{[1] T}\right)\right) A^{[1] T}\right)_{i i}}{k_{\text {out }}^{[1]}(i)\left(k_{\text {out }}^{[1]}(i)-1\right)\left(1-c_{\text {out }}^{[1]}(i)\right)}$
$\left(k_{\text {in }}^{[1]}(i) k_{\text {out }}^{[1]}(i)-\left(A^{[1]}\right)_{i i}^{2}+0.5 k_{\text {out }}^{[1]}\left(k_{\text {out }}^{[1]}-1\right)\right)\left(1-c_{\text {both }}^{[1]}(i)\right)$

not included here for brevity), indicate that transitions between synchronous and asynchronous regions of parameter space are associated with corresponding transitions in the dynamical regime of (1) - in particular, critical behaviour is associated with high frequency and/or amplitude oscillation. Our results therefore reflect the divergence of anatomy and the functional network configurations that they support [41]; moreover, they indicate that structurefunction relations at, and near, criticality are strongly dependent on specific neural dynamics (in contrast with a growing consensus $[25,40,42,43]$ that structure-function relations can be understood predominantly in terms of criticality) and that this is especially true in the case of emergent functional connectivity and of more weakly synchronous neural activity.

In Figures 4(d-f) we highlight the variability in network structure for three different fixed values of the basal input parameter pair $(P, Q)$ (as evidenced by clustering) and the local differences between $C(i)$ and $\widetilde{C}^{\text {both }}(i)$. We observe strong inter-node variation and, moreover, that even for low overall clustering particular network areas show very different properties. Comparison of $C(i)$ and $\widetilde{C}^{\text {both }}(i)$ shows similar trends, although zeros exist in $\widetilde{C}^{\text {both }}(i)$ that are not reflected in $C(i)$. One of these is common across Figures $4(\mathrm{~d}-\mathrm{f})$ and reflects the local clustering of the underlying structural layer; the remainder arise due to synchronisation behaviour.

Conclusion. - In this letter, we have considered the application of multiplex network models to shed light on the emergent structure-function relations in neural systems that are fundamental to the wide functional repertoire of higher brain functions. This work provides the first demonstration that a simultaneous analysis of structurefunction networks is better placed to capture emergent features and capabilities of neural systems than standard single-layer analyses.

Our results indicate that (in common with $[25,40,42]$ ), increased structure-function relations evidenced by multiplex measures of overlap and clustering were associated 


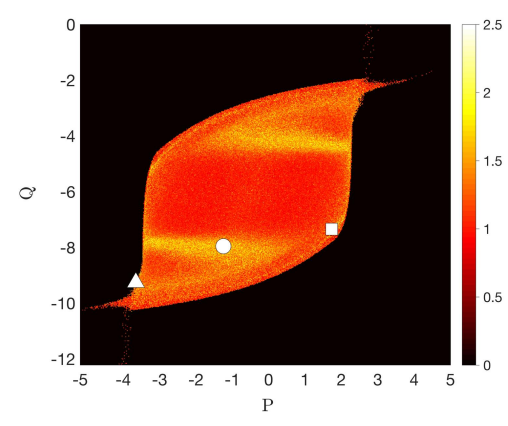

(a)

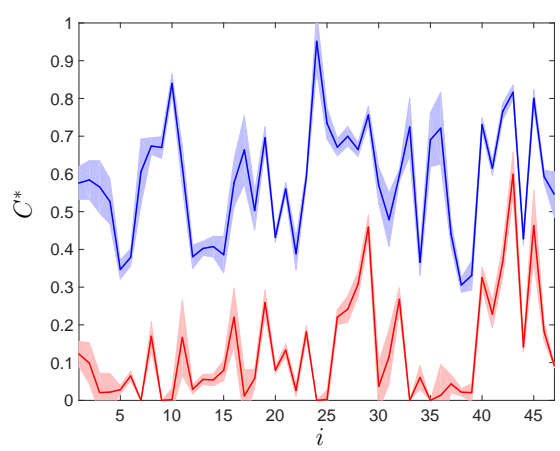

(d)

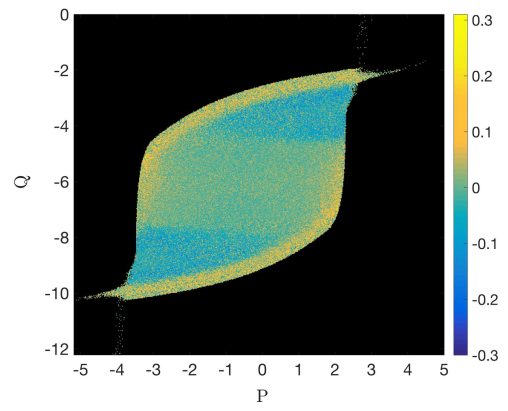

(b)

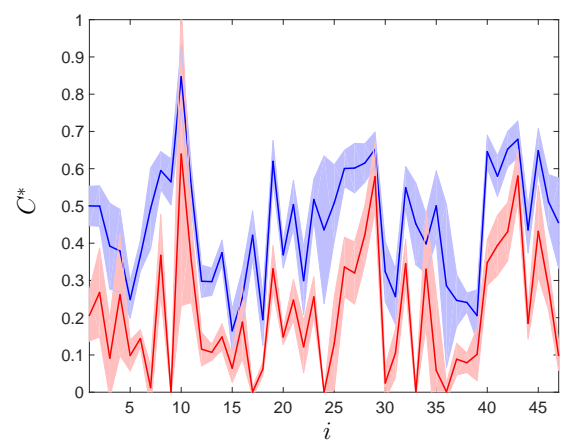

(e)

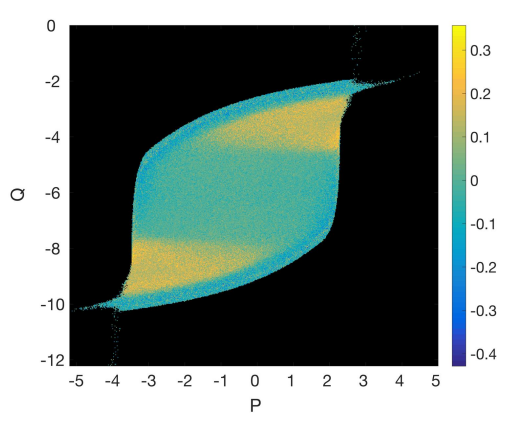

(c)

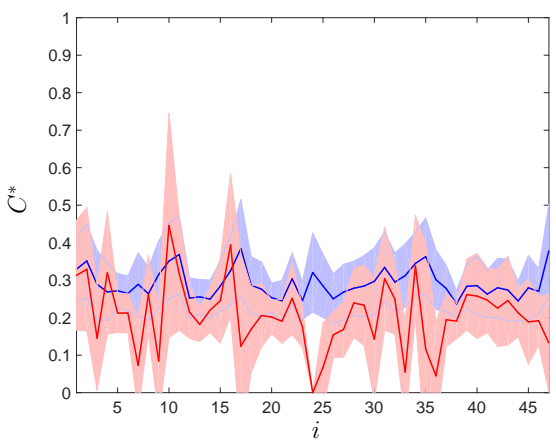

(f)

Fig. 4: Multiplex clustering coefficients corresponding to the neurologically relevant patterns described in Table 1. (a) Variation of $\widetilde{\mathcal{C}}^{\text {both }} /\left\langle\widetilde{\mathcal{C}}_{\text {rand }}^{\text {both }}\right\rangle$ as a function of the basal input parameters $P$ and $Q$. (b),(c) The contribution of key network motifs to the clustering $\widetilde{\mathcal{C}}^{\text {both }}$ expressed via $\widetilde{\mathcal{C}}^{\text {both }} /\left\langle\widetilde{\mathcal{C}}_{\text {rand }}^{\text {both }}\right\rangle-\widetilde{\mathcal{C}}^{\#} /\left\langle\widetilde{\mathcal{C}}_{\text {rand }}^{\#}\right\rangle$ for (b) \#= cyc, (c) \#= out. (d)-(f) Comparison of local standard multiplex clustering $C(i)$ (shown in blue) and $\widetilde{C}^{\text {both }}(i)$ (shown in red) for specific parameter values $(P, Q)=(-3.5,-9.1)$, $(-1.1,-7.8)$ and $(1.8,-7.2)$ highlighted in (a) by a triangle, circle and square, respectively. The shaded region indicates a confidence interval of width one standard deviation.

with criticality of the underlying neural model. However, the detail of this relation differed significantly to that obtained from single-layer measures. Moreover, by employing a novel multiplex measure, designed specifically to accommodate the divergence between anatomical brain networks and the functional behaviour they support, this work supports a deeper theoretical understanding of the neural substrates underlying cognition. Our results highlight that the form of structure-function relations differs significantly, depending on the specific dynamical regime of the neural system, thereby suggesting a more subtle dependence of network behaviour than one dominated by criticality alone.

Important future work will incorporate more general multilayered aspects of neural systems, including consideration of the transient nature of various dynamical brain states, in the form of time-varying multiplex structures, for example. Such studies will have relevance to neurological disorders (such as epilepsy, Alzheimer's disease and other dementias), potentially providing additional insight to single layer analyses (see see e.g. $[44,45]$ for discussion of the clinical applications of network science) that have evidenced altered patterns of connectivity in both struc- ture and function across a broad range of brain disorders.

$$
* * *
$$

Multiplex network images were generated using MuxViz $[46]$.

\section{REFERENCES}

[1] Newman M. E. J., Networks: An Introduction (Oxford University Press) 2010.

[2] Barahona M. and Pecora L. M., Phys. Rev. Lett., 89 (2002) 054101.

[3] Wu Y., Shang Y., Chen M., Zhou C. and Kurths J., Chaos, 18 (2008) 037111.

[4] Wang X. F. and Chen G., IEEE Trans. Circuits Syst. I: Fundam. Theory Appl., 49 (2002) 54.

[5] O'Dea R., Crofts J. J. and Kaiser M., J. R. Soc. Interface, 10 (2013) 20130016.

[6] Zhou C., Zemanová L., Zamora G., Hilgetag C. C. and Kurths J., Phys. Rev. Lett., 97 (2006) 238103.

[7] Lameu E. L., Borges F. S., Borges R. R., Iarosz K. C., Caldas I. L., Batista A. M., Viana R. L. and Kurths J., Chaos, 26 (2016) 043107. 
[8] Hlinka J. and Coombes S., Eur. J. Neurosci., 36 (2012) 2137.

[9] Henderson J. and Robinson P., Brain connect., 4 (2014) 112.

[10] Lo Y.-P., O’Dea R., Crofts J. J., Han C. E. and Kaiser M., Sci. Rep., 5 (2015) 15397.

[11] Sporns O., Networks of the Brain (The MIT Press) 2010.

[12] Bassett D. S., Meyer-Lindenberg A., Achard S., Duke T. and Bullmore E., Proc. Natl. Acad. Sci., 103 (2006) 19518.

[13] van den Heuvel M. P. and Sporns O., Trends Cogn. Sci., 17 (2013) 683.

[14] Meunier D., Lambiotte R. and Bullmore E. T., Front. Neurosci., 4 (2010) 200.

[15] van den Heuvel M. P., Mandl R. C., Stam C. J., Kahn R. S. and Pol H. E. H., J. Neurosci., 30 (2010) 15915.

[16] Crofts J. J., Higham D. J., Bosnell R., Jbabdi S., Matthews P. M., Behrens T. and Johansen-Berg H., NeuroImage, 54 (2011) 161.

[17] Batalle D., Eixarch E., Figueras F., Muoz-Moreno E., Bargallo N., Illa M., Acosta-Rojas R., AmatRoldan I. and Gratacos E., NeuroImage, 60 (2012) 1352 .

[18] Cabral J., Fernandes H. M., Van Hartevelt T. J., James A. C., Kringelbach M. L. and Deco G., Chaos, 23 (2013) 046111.

[19] Stam C., Jones B., Nolte G., Breakspear M. and Scheltens P., Cerebral Cortex, 17 (2007) 92.

[20] Supekar K., Menon V., Rubin D., Musen M. and Greicius M. D., PLoS Comput. Biol., 4 (2008) e1000100.

[21] Liu Y., Liang M., Zhou Y., He Y., Hao Y., Song M. Yu C., Liu H., Liu Z. and Jiang T., Brain, 131 (2008) 945.

[22] Chavez M., Valencia M., Navarro V., Latora V. and Martinerie J., Phys. Rev. Lett., 104 (2010) 118701.

[23] PARK H.-J. and Friston K., Science, 342 (2013) 1238411.

[24] Wang Z., Dai Z., Gong G., Zhou C. and He Y., Neuroscientist, 21 (2015) 290.

[25] Stam C., Straaten E. V., Dellen E., Tewarie P., Gong G., Hillebrand A., Meier J. and Mieghem P., Int. J. Psychophys., 103 (2016) 149.

[26] Bianconi G., Phys. Rev. E, 87 (2013) 062806.

[27] Battiston F., Nicosia V. and Latora V., Phys. Rev.
E, 89 (2014) 032804.

[28] Cozzo E., Kivelä M., De Domenico M., SoléRibalta A., Arenas A., Gómez S., Porter M. A. and Moreno Y., New J. Phys., 17 (2015) 073029.

[29] Kötter R., Neuroinformatics, 2 (2004) 127.

[30] Wilson H. R. and Cowan J. D., Biophys. J., 12 (1972) 1.

[31] Hoppensteadt F. C. and Izhikevich E. M., Weakly connected neural networks Vol. 126 of Appl. Math. Sci. (Springer Science \& Business Media) 1997.

[32] Jansen B. H. and Rit V. G., Biol. Cybernet., 73 (1995) 357.

[33] Grimbert F. and Faugeras O., Neural Comput., 18 (2006) 3052.

[34] Liley D. T., Bojak I., Dafilis M. P., van Veen L., Frascoli F. and Foster B. L., Bifurcations and state changes in the human alpha rhythm: theory and experiment in Modeling Phase Transitions in the Brain (Springer) 2010 pp. 117-145.

[35] Mormann F., Lehnertz K., David P. and Elger C. E., Physica D: Nonlinear Phenom., 144 (2000) 358.

[36] Fagiolo G., Phys. Rev. E, 76 (2007) 026107.

[37] Gollo L. L. and Breakspear M., Phil. Trans. R. Soc. $B, 369$ (2014) 20130532.

[38] Haimovici A., Tagliazucchi E., Balenzuela P. and Chialvo D. R., Phys. Rev. Lett., 110 (2013) 178101.

[39] Yu S., Yang H., Shriki O. and Plenz D., Front. Syst. Neurosci., 7 (2013) 42.

[40] Deco G., Ponce-Alvarez A., Mantini D., Romani G., Hagmann P. and Corbetta M., J. Neurosci., 33 (2013) 11239.

[41] Mišić B., Betzel R. F., de Reus M. A., van den Heuvel M. P., Berman M. G., MeIntosh A. R. and Sporns O., Cereb. Cortex, 26 (2016) 3285.

[42] Rubinov M., Sporns O., Thivierge J.-P. and BreakSPEAR M., PLoS Comput. Biol., 7 (2011) e1002038.

[43] Haimovici A., Tagliazucchi E., Balenzuela P. and Chialvo D. R., Phys. Rev. Lett., 110 (2013) 178101.

[44] Bassett D. S. and Bullmore E. T., Curr. Opin. Neurol., 22 (2009) 340.

[45] Sporns O., Dialog. Clin. Neurosci., 15 (2013) 247.

[46] De Domenico M., Porter M. A. and Arenas A., J. Complex Netw., (2014) cnu038. 